\title{
Les traitements médicaux de l'oligoasthénozoospermie idiopathique
}

Revue de la littérature

\author{
A. Demoulin
}

Université de Liège, Centre de Procréation Médicalement Assistée, CHR-Citadelle, 1 Bd du 12ème de Ligne, 4000 Liège, Belgique.

\section{RESUME}

Dans un nombre élevé de cas, l'infertilité d'un couple résulte d'une oligoasthénozoospermie dont aucune étiologie ne peut être reconnue. Différentes thérapeutiques médicales ont été proposées dans la littérature pour accroître la numération et la mobilité des spermatozoïdes. Nombre d'études observent une amélioration des paramètres spermiologiques sans prendre en compte les variations physiologiques et sans les comparer à des patients traités par un placebo. Pour d'autres, le but final du traitement n'est pas précisé c'est-à-dire l'obtention d'un enfant par le couple.

Dans cette revue de la littérature, seules les études randomisées précisant les taux de grossesses ont été prises en compte. Différentes hormones de l'axe hypothalamo-hypophyso-testiculaire ont été utilisées, le GnRH et ses analogues, les gonadotrophines et leurs formes purifiées, les anti-estrogènes, les androgènes et les inhibiteurs de l'aromatisation. Parmi ces molécules, des effets positifs ont été sporadiquement observés. Ainsi, la mestérolone stimulerait la spermatogenèse dans une étude sur cinq. L'undécanoate de testostérone et la FSH auraient des effets bénéfiques sur le pouvoir fécondant in vitro. Deux anti-estro- gènes ont été testés, le clomifène et le tamoxifène avec des taux de grossesse significativement augmentés mais cependant des effets paradoxaux ont été observés. D’autres molécules, non hormonales, ont également fait l'objet de travaux. La plus étudiée est la kallicréine, une glycoprotéine impliquée dans la libération des kinines à partir du kininogène. Des résultats contradictoires ont été obtenus avec cette thérapie. La pentoxifylline, un inhibiteur de la phosphodiestérase est active in vitro mais n'a pas encore fait ses preuves in vivo. D'autres substances comme l'arginine, la carnitine, les $\alpha$ bloqueurs, les bloqueurs des mastocytes, l'acide folinique, les vitamines, etc, font l'objet de publications sporadiques et aucume conclusion ne peut être tirée.

En conclusion, l'oligoasthénozoospermie idiopathique semble toujours rebelle à la plupart des traitements dont nous disposons à ce jour. Dans ce cas, le choix d'une thérapie est empirique et devrait en cas d'échec être rapidement abandonné afin d'orienter le couple vers la procréation médicalement assistée.

Mots clés: Infertilité masculine, idiopathique, traitements médicaux. 
Lorsque l'on est confronté à un problème d'oligo- et/ou asthéno-zoospermie chez un patient infertile, l'anamnèse, l'examen clinique, la biologie, et la spermoculture s'avèrent souvent négatifs. Ainsi, Baker et al [7] trouvent près de $80 \%$ d'étiologie idiopathique sur une série de plus de mille cas. Se pose alors le problème d'un traitement de cette infertilité. Le développement des techniques de procréation médicalement assistée a certainement apporté la solution à un certain nombre de ces cas. Mais n'existe-t-il pas des traitements médicaux capables de stimuler la spermatogenèse et la mobilité des spermatozoïdes pour tenter de traiter ces patients, plutôt que de les entraîner vers des techniques qu'ils ne sont pas toujours prêts à accepter?

Sur la base des mécanismes endocriniens et biochimiques de la régulation de la fonction testiculaire et épididymaire, différents traitements ont été proposés depuis quelques décennies. Dans ce travail, nous avons tenté d'analyser les résultats obtenus avec les hormones ou des médiateurs biochimiques.

Les problèmes relevés lors de cette analyse sont de trois ordres:

1. Les critères de choix des patients acceptés dans l'étude. Ainsi, on voit que l'oligospermie a des définitions différentes selon le temps et les auteurs (20 à $40 \times 10^{6}$ spermatozoïdes $/ \mathrm{ml}$ ).

2. La durée et les doses administrées varient d'une étude à l'autre. Un des reproches les plus importants que l'on peut formuler est l'absence d'un groupe contrôle comparatif.

3. Un manque de systématisation dans l'analyse des résultats étudiés. Si certains auteurs constatent un effet bénéfique lorsqu'ils observent une amélioration des qualités du spermogramme, d'autres plus finalistes considèrent comme un succès du traitement la survenue d'une grossesse. Malheureusement, le délai d'obtention de cette grossesse après l'instauration et/ou l'arrêt du traitement n'est que rarement précisé.

Dans ce travail, nous avons retenu les articles réalisés avec un groupe contrôle et prenant comme critère d'efficacité d'un traitement, l'apparition d'une grossesse chez la partenaire. Différentes hormones ont été testées. Elles ont un site d'action hypothalamo-hypophysaire ou testiculaire.

\section{Les hormones hypothalamiques:}

Le LHRH administré de façon pulsatile chez des patients atteints d'oligospermie avec gonadotrophines normales a fait l'objet de très peu d'études, et ne semble pas efficace $[2 ; 21]$. Crottaz et al [19], dans une étude en double aveugle, n'observent pas d'augmentation des qualités du spermogramme ni du taux de grossesse, avec le LHRH, de même que Schwaerztein et Aparicio [48] ne peuvent conclure à un effet bénéfique des analogues.

\section{Les hormones hypophysaires:}

1. Les gonadotrophines: L'hormone chorionique gonadotrope HCG (Pregnyl ${ }^{\circledR}$ Profasy ${ }^{\circledR}$ ) a été utilisée pendant de nombreuses années pour son effet stimulant la production de testostérone par les cellules de Leydig. Souvent, ce traitement était associé à de l'hormone follicule stimulante (HMG, Humegon $\odot$, Menogon) FSH urinaire (Metrolin ${ }^{\circledR}$, Metodin $\left.\mathrm{HP}^{\circledR}\right)$, ou recombinante $\left(\right.$ Gona $/ \mathrm{F}^{\circledR}$ - Puregon $\left.{ }^{\circledR}\right)$. Dans des études non contrôlées, une amélioration des paramètres du spermogramme était fréquemment observée $[2 ; 8]$. Cependant, une étude avec groupe témoin n'a pas confirmé cet effet thérapeutique, le taux de grossesse étant comparable dans les deux groupes [40]. En revanche, l'utilisation de FSH purifiée à doses élevées serait génératrice d'une amélioration des paramètres du sperme [10; 27]. Selon Acosta [3], la FSH recombinante améliorerait l'ultrastructure des spermatozoïdes et serait responsable d'un accroissement du taux de fécondation des ovocytes in vitro. Aucune étude multicentrique in vivo n'a cependant été publiée à ce jour.

2. L'hormone de croissance: Comme chez la femme, l'hormone de croissance a été impliquée dans le processus de la gamètogenèse. Cette hormone, sous sa forme recombinante, a été testée dans l'oligoasthénospermie idiopathique, sans obtenir de succès notoire $[4 ; 32]$, sauf peut-être dans l'asthénozoospermie: $33 \%$ 
de grossesses contre $0 \%$ chez les oligozoospermiques [37].

\section{Les inhibiteurs de la prolactine:} Puisque les perturbations de la spermatogenèse accompagnées d'une hyperprolactinémie sont habituellement traitées par la bromocriptine, l'idée de donner ce traitement aux cas idiopathiques a été tentée. Trois études comparant les résultats avec ceux obtenus avec un placebo montrent que ce traitement est inefficace dans ces conditions (Tableau 1). Dans la méta-analyse réalisée par Vandekerkhove et al [56] ou O'Donovan et al [36], les résultats combinés montrent l'absence de différence entre les patients traités et ceux ayant reçu un placebo, en terme de grossesses obtenues.

\section{Les antiestrogènes:}

Deux molécules, le clomifène $\left(\right.$ Clomid ${ }^{\circledR}$, Pergotine ${ }^{\circledR}$ ) et le tamoxifène (Nolvadex ${ }^{\circledR}$, Tamoplex $\left.{ }^{\circledR}\right)$ sont des antiestrogènes se fixant au niveau des récepteurs hypothalamo-hypophysaires. Ils induisent une augmentation des gonadotrophines qui agissent à leur tour sur le testicule. Ils ont fait l'objet de nombreuses publications non contrôlées qui ont conclu à une action bénéfique sur l'un ou l'autre des paramètres du sperme et sur l'obtention de grossesses $[44 ; 15 ; 21]$. Cependant, les études comparatives contre un placebo furent nettement moins éloquentes. Bien que statistiquement, lorsqu'on rassemble tous les travaux, on observe une augmentation significative du taux de grossesse dans le groupe des patients traitées par Clomifène (Tableau 2) ou par Tamoxifène (Tableau 3) par rapport aux patients traitées par un placebo. Il faut cependant remarquer que la plupart des études prises séparément n'ont pas observé de variation significative et que Glass [22] relate trois cas d'effets paradoxaux du Tamoxifène, avec chute de la numération et de la mobilité après traitement.

\section{Les androgènes:}

Certaines étapes de la spermato-et/ou de la spermio-genèse sont sous la dépendance de la testostérone, secrétée par les cellules de Leylig. Il paraissait donc logique de tenter des traitements androgéniques. Différents androgènes sont proposés par les firmes pharmaceutiques: la mestérolone (Proviron ${ }^{\circledR}$ ) l'énanthate de testostérone (Testoviron $\left.{ }^{\circledR}\right)$, l'undécanoate de testostérone (Undestor ${ }^{\circledR}$ ), l'androstanolone (Andractim $\left.{ }^{\circledR}\right)$, la méthyltestostérone ... Certains sont hépatotoxiques et la plupart ne pénètre pas dans le compartiment intratesticulaire à l'exception de la mestérolone [2]. Tous ces traitements n'ont pas fait l'objet d'études systématiques.

Certains ont été utilisés dans le but d'un "rebound effect". Sous androgène à dose élevée, une oligospermie sévère, voire une azoospermie s'installe, et à l'arrêt du traitement, un effet récupérateur apparait parfois. Vu le nombre d'échecs, ce traitement est actuellement abandonné. La mestérolone a fait l'objet d'un certain nombre d'études versus contrôle (Tableau 4). Toutes se sont montrées inactives en terme de grossesse sauf l'étude de Varma et al, [57].

Trois études ont utilisé l'undécanoate de testostérone $[40 ; 16]$, une seule s'est avérée efficace [24]. En FIVETE, Comhaire et al [17] ont montré une augmentation du taux de fécondation identique à celui observé avec un placebo.

L'utilisation d'inhibiteurs de l'aromatase qui empêchent la transformation de la testostérone en estradiol a été essayée. Si le traitement par Testolactone paraissait attrayant, Clark and Sherins [14], dans une étude contrôlée, furent incapables de voir une amélioration du sperme et du taux de grossesse.

D'autres molécules furent également l'objet d'études dans la littérature:

1. La kallicréine (Padutin ${ }^{\circledR}$ ) est une enzyme responsable de la transformation du kininogène en kinines; ces dernières molécules s'étaient montrées actives sur la spermatogenèse et sur la mobilité des spermatozoïdes. Après un nombre important de publications non contrôlées vantant l'effet bénéfique de la kallicréine sur les qualités du spermogramme [46], les travaux comparant l'effet de la molécule contre un placebo sont apparus dans la littérature (Tableau 5). Deux études sur six confirmèrent un taux de grossesse plus favorable sous traitement rendant l'étude significative. 
Tableau 1 : Traitements medicaux de l'oas idiopathique bromocriptine

\section{ETUDES CONTROLEES}

\begin{tabular}{lccccc}
\hline Auteurs & Année & Nombre de cas & $\begin{array}{c}\text { Grossesses } \\
\text { Traitement }\end{array}$ & $\begin{array}{c}\text { Grossesses } \\
\text { Placebo }\end{array}$ & P \\
\hline HOVATTA et al [26] & 1979 & 40 & $1 / 20$ & $2 / 18$ & NS \\
AINMELK et al [5] & 1982 & 12 & $0 / 6$ & $0 / 6$ & NS \\
LUNGLMAYR et al [33] & 1983 & 50 & $2 / 25$ & $2 / 25$ & NS \\
\hline TOTAL & & & $\mathbf{3 / 5 1}$ & $\mathbf{4 / 4 9}$ & \\
& & & $\mathbf{5 . 9 \%}$ & $\mathbf{8 . 1 \%}$ \\
\hline
\end{tabular}

Tableau 2 : Traitements medicaux de l'oas idiopathique citrate de clomifene

ETUDES CONTROLEES

\begin{tabular}{lccccc}
\hline Auteurs & Année & Nombre de cas & $\begin{array}{c}\text { Grossesses } \\
\text { Traitement }\end{array}$ & $\begin{array}{c}\text { Grossesses } \\
\text { Placebo }\end{array}$ & P \\
\hline WIELAND et al [62] & 1972 & 21 & $1 / 11$ & $\mathbf{1} 10$ & NS \\
PAULSON [38] & 1979 & 40 & $2 / 20$ & $7 / 20$ & NS \\
RONNBERG [43] & 1980 & 29 & $1 / 14$ & $\mathbf{1 / 1 5}$ & NS \\
Scottish Infertility Group [49] & 1982 & 179 & $15 / 93$ & $\mathbf{1 0 / 8 6}$ & NS \\
WANG et al [58] & 1983 & 36 & $4 / 29$ & $0 / 7$ & NS \\
MICIC et DOTLIC [35] & 1985 & 101 & $7 / 85$ & $0 / 45$ & 0,02 \\
SOKOL et al [52] & 1988 & 20 & $\mathbf{1 / 1 1}$ & $4 / 9$ & NS \\
ROGERS et al [42] & 1988 & 36 & $5 / 18$ & $0 / 18$ & 0,02 \\
CHECK et al [13] & 1989 & 100 & $29 / 50$ & $8 / 50$ & 0,001 \\
WHO [61] & 1992 & 141 & $7 / 70$ & $6 / 71$ & NS \\
BREZNIK et BORKO [12] & 1993 & 47 & $3 / 20$ & $8 / 27$ & NS \\
\hline & & & $\mathbf{7 5 / 3 7 4}$ & $\mathbf{4 5 / 3 4 0}$ & $\mathbf{2 0 \%}$ \\
\hline
\end{tabular}


Tableau 3 : Traitements medicaux de l'oas idiopathique tamoxifene

ETUDES CONTROLEES

\begin{tabular}{lccccc}
\hline Auteurs & Année & Nombre de cas & $\begin{array}{c}\text { Grossesses } \\
\text { Traitement }\end{array}$ & $\begin{array}{c}\text { Grossesses } \\
\text { Placebo }\end{array}$ & P \\
TOROK [54] & 1985 & 54 & $9 / 27$ & $5 / 27$ & NS \\
HARGREAVE et al [25] & 1986 & 80 & $8 / 36$ & $10 / 44$ & NS \\
AINMELK et al [4] & 1987 & 32 & $2 / 16$ & $0 / 16$ & NS \\
ROGERS et al [42] & 1988 & 36 & $7 / 18$ & $0 / 18$ & 0,01 \\
KRAUSE et al [31] & 1992 & 76 & $5 / 39$ & $3 / 37$ & NS \\
BREZNIK et BORKO [12] 1993 & 49 & $7 / 22$ & $8 / 27$ & NS \\
\hline
\end{tabular}

Tableau 4 : Traitements medicaux de l'oas idiopathique mesterolone

ETUDES CONTROLEES

\begin{tabular}{lccccc}
\hline Auteurs & Année & Nombre de cas & $\begin{array}{c}\text { Grossesses } \\
\text { Traitement }\end{array}$ & $\begin{array}{c}\text { Grossesses } \\
\text { Placebo }\end{array}$ & P \\
\hline AAFJES et al [1] & 1983 & 53 & $4 / 27$ & $5 / 32$ & NS \\
WANG et al [59] & 1983 & 19 & $0 / 12$ & $0 / 7$ & NS \\
Scottish Infertility Group [50] & 1984 & 328 & $34 / 176$ & $28 / 152$ & NS \\
VARMA et PATEL [57] & 1988 & 350 & $115 / 250$ & $14 / 100$ & 0,001 \\
WHO [60] & 1989 & 105 & $15 / 69$ & $5 / 36$ & NS \\
GERRIS et al [20] & 1991 & 52 & $7 / 27$ & $\mathbf{1 2 / 2 5}$ & NS \\
\hline & & & $\mathbf{1 6 8 / 5 3 4}$ & $\mathbf{5 2 / 3 2 7}$ & $\mathbf{1 6 \%}$ \\
\end{tabular}


Tableau 5 : Traitements medicaux de l'oas idiopathique kallicréine

ETUDES CONTROLEES

\begin{tabular}{lccccc}
\hline Auteurs & Année & Nombre de cas & $\begin{array}{c}\text { Grossesses } \\
\text { Traitement }\end{array}$ & $\begin{array}{c}\text { Grossesses } \\
\text { Placebo }\end{array}$ & P \\
\hline SCHILL [45] & 1979 & 90 & $14 / 48$ & $5 / 42$ & 0,05 \\
BEDFORD et ELSTEIN [9] & 1981 & 23 & $1 / 13$ & $1 / 10$ & NS \\
IZZO et al [28] & 1984 & 29 & $2 / 15$ & $0 / 14$ & NS \\
MICIC et al [34] & 1985 & 75 & $9 / 45$ & $0 / 30$ & 0,01 \\
KECH et al [29] & 1994 & 91 & $4 / 44$ & $4 / 47$ & NS \\
\hline & & & $\mathbf{3 0 / 1 6 5}$ & $\mathbf{1 0 / 1 4 3}$ & \\
& & & $\mathbf{1 8 . 2 \%}$ & $\mathbf{7 \%}$ &
\end{tabular}

2. La pentoxifylline (Torental $\left.{ }^{\circledR}\right)$ est un dérivé de la méthylxanthine qui agit après administration orale en améliorant la microcirculation notamment au niveau du testicule et de l'épididyme, favorisant l'action androgénique au sein de ces organes. $\mathrm{Si}$, in vitro, la mobilité des spermatozoïdes et leur pouvoir fécondant semblent améliorés, l'effet n'est pas comparable en fécondation naturelle. Deux études contrôlées ne confirment pas en effet cette stimulation de la mobilité ni l'augmentation du taux de grossesse [59; 55].

3. D’autres médicaments ont également été testés, mais par un nombre d'auteurs beaucoup plus limité. Les bloqueurs des mastocytes $\left\{\right.$ Tranislast. Riaben ${ }^{\circledR}$ (Japon) - Kétotifène Zaditène (Belgique)\} ont été étudiés par Yamamoto et al [64] et Schill et al [47] respectivement. Les mastocytes seraient en effet présents dans les testicules de patients infertiles. Le groupe japonais a observé un taux de grossesse de $28 \%$ dans le groupe de patients traités par rapport à $0 \%$ chez les patients ayant reçu un placebo.

L'utilisation des $\alpha$ bloquants a été testée pour son effet dilatateur sur les tubules épididymaires. Une étude récente de Yamamoto et al [63] montre un effet bénéfique sur la numération, mais le taux de grossesse (25\%) n'a pas été significativement modifié par rapport au groupe contrôle $(6,7 \%)$.
Une étude multicentrique italienne [18], ainsi que Vitali et al [58] ont recherché l'influence de la L-carnitine sur l'asthénospermie. Ils observent un accroissement de la mobilité mais l'étude n'était pas contrôlée et le taux de grossesse n'est pas rapporté. Des études complémentaires paraissent indispensables pour confirmer cette observation qui pourrait être intéressante. Des traitements par acides aminés ont également été essayés et notamment l'arginine. Pryor et al [39] avaient conclu à une inactivité, alors que Aydan et al [6] ont obtenu $20 \%$ de grossesses dans une étude sans groupe contrôle.

Les vitamines ont été testées. La vitamine $\mathbf{E}$, agissant comme antioxydant pour traiter les espèces oxygéno-réactives, semble se montrer active puisque $21 \%$ des sujets asthénospermiques traités ont obtenu une grossesse, alors qu'aucune n'a été obtenue dans le groupe placebo [53]. L'acide folinique a été utilisé dans les infertilités masculines avec syndrome de cellules rondes idiopathiques avec succès dans $37 \%$ des cas, malheureusement sans comparaison avec un groupe contrôle [11].

L'acupuncture s'est également révélée efficace (30\% de grossesses) en agissant essentiellement sur la nécrospermie et l'asthénozoospermie [51]. Ce travail mérite certainement une vérification et une étude plus approfondie. 
En conclusion, il ressort de cette analyse non exhaustive de la littérature que les traitements médicaux de l'oligospermie idiopathique restent empiriques. Parmi les traitements hormonaux, seuls les antiestrogènes (tamoxifène $2 \mathrm{mg} / \mathrm{j}$ clomifêne $25-50 \mathrm{mg} / \mathrm{j}$ ) semblent augmenter le taux de grossesse dans les études contrôlées. La méta analyse portant sur la mesterolone (150 mg/j) paraît également montrer un effet bénéfique dans les oligoasthénozoospermies modérées. La kallicréine $(\mathrm{mg} / \mathrm{j})$ paraissait également active, mais ces résultats semblent contredits actuellement [23].

Il apparaît également surprenant que de nos jours, autant de pathologies du sperme soient encore cataloguées d'idiopathiques. Une réflexion multidisciplinaire devrait être mise en place afin de tenter d'établir une étiologie à ces pathologies. Encore faudrait-il savoir si un traitement efficace serait à la disposition du patient pour rétablir sa fertilité et lui éviter le passage aux procréations médicalement assistées. Une approche étiologique permettrait indubitablement un meilleur choix thérapeutique, mais les couples qui nous consultent auront-ils encore la patience d'attendre un éventuel résultat avant d'envisager le passage à l'injection intracytoplasmique des quelques spermatozoïdes que nous trouvons dans leur éjaculation puisque d'excellents résultats sont obtenus avec cette technique.

\section{REFERENCES}

1. AAFJES J., VAN DER VIJVER J., BRUGMAN F. \& SCHENK P. Double-blind cross over treatment with mesterolone and placebo of subfertile oligozoospermic men-value of testicules biopsy. Andrologia, 1983, 15 : 531-535.

2. ACOSTA A.A. Pharmacologic improvement of the sperm function: treatment of the patient. In J. Tesarick ed. Male Factor in human infertility. Frontiers in Endocrinology vol 8. Ares sereno symposia, 1994 : 217-255.

3. ACOSTA A.A., KHALIFA E. \& OEHNINGER S. Pure human follicle-stimulating hormone has a role in the treatment of severe male infertility by assisted reproduction: Norfolk's total experience. Human Reprod., 1992, $7: 1067-1072$.

4. AINMELK Y., BELISLE S., CARMEL M. \& TETREAULT L. Tamoxifen citrate therapy in male infertility. Fertil. Steril., 1987, 48 : 113-117.
5. AINMELK Y., BELISLES S., KANDALAFT N., Mc CLURE D., TETREAULT L. \& ELMILALI M. Bromocriptine therapy in oligozoospermic infertile men. Arch. Androl., 1982, 8 : 135-141.

6. AYDAN S., INCI O. \& ALAGOL B. The role of argenin, indomethacin and kallikrein in the treatment of oligoasthenospermia. Int. Urol. and Nephrol., 1995, $27: 199-202$.

7. BAKER H.W.G., BURGER H.G., de KRETSER D.M. \& HUDSON B. Relative incidence of etiological disorders in male infertility. In: Santen R.J. \& Swerdloff R.S. eds. Male Reproductive Dysfunction. New York, Marcel Dekker, USA, 1986, 341-372.

8. BARTOOV B., ELTES F., LUNENFELD E., HAREVEN D., LEDERMAN H. \& LUNENFELD B. Sperm quality of subfertile male before and after treatment with human follicle-stimulating hormone. Fertil. Steril., 1994, 61 : 727-734.

9. BEDFORD N. \& ELSTEIN M. The effect of kallikrein in male infertility: a double blind study. In: Insler V. \& Bettendorf G. eds. Diagnosis and Treatment of infertility. Elsevier, Amsterdam, 1981 : 339-344.

10. BENNET A. Place des traitements par FSH dans les stérilités masculines. Contracept. Fertil. Sex., 1992, 20 : 705-707.

11. BENTIVOGLIO G., MELICA F. \& CRISTOFORONI P. Folinic acid in the treatment of human male infertility. Fertil. Steril., 1993, $60: 698-701$.

12. BREZNIK R. \& BORKO E. Effectiveness of antiestrogens in infertile men. Archives of Andrology, 1993, 31 : 43-48.

13. CHECK J., CHASE J., NOWROOZI K., WU C. \& ADELSON M. Empirical therapy of the male with clomiphen in couple with unexplained infertility. Int. J. Fertil., 1989, $34: 120-122$.

14. CLARK R. \& SHERINS R. Clinical trial of testolactone for treatment of idiopathic male infertility. J. Androl., 1989, $10: 240-247$.

15. COMHAIRE F.H. Tamoxifen. In: Bain J., Schill W.P. \& Schwarzstein L. eds. Treatment of male infertility. Springer Verlag, Berlin, $1982:$ 45-53.

16. COMHAIRE F.H. Treatment of idiopathic testicular failure with high dose testosterone undecanoate a double blind pilot study. Fertil. Steril., 1990, 54 : 689693.

17. COMHAIRE F.H., SCHOONJANS F., ABDELMASSIH R.et al. Does treatment with testosterone undecanoate improve the in vitro fertilizing capacity of spermatozoa in patients with idiopathic testicular failure? (results of a double blind study). Hum. Reprod., 1995, $10: 2600-2602$.

18. COSTA M., CANALE D., FILICORI M., D'IDDIO S. \& LENZI A. L-Carnitine in idiopathic asthenozoospermia: a multicenter study. Andrology, 1994, 26 : 155-159. 
19. CROTTAZ B., SENN A., REYMOND M.J., REY F., GERMOND M., GOMEZ F. Follicle stimulating hormone bioactivity in idiopathic normagonadotropic olidoasthenozoospermia: double blind trial with gonadotropin-releasing hormone. Fertil. Steril., 1992, 57 : 1034-1043.

20. GERRIS J., COMHAIRE F., HELLEMANS P., PEETERS K., SCHOONJANS F. Placebo-controlled trial of high-dose mesterolone treatment of idiopathic male infertility. Fertil. Steril., 1991,55 : 603-607.

21. GILBAUGH J.H. \& LIPSHULTZ L.I. Non surgical treatment of male infertility. Urologic Clinics of North America, 1994 : 21, 531-548.

22. GLASS A.R. Paradoxical suppression of spermatogenesis during tamoxifen treatment of oligospermia. Fertil. Steril., 1988, 49 : 376-377.

23. GLEZERMAN M., LUNENFELD E., POTASHNIK G., HULEINEL M., SOFFER Y. \& SEGAL S. efficacy of kallicrein in the treatment of oligozoospermia and asthenozoospermia: a double blind trial. Fertil. Steril., 1993, 60 : 1052-4056.

24. GREGORIOU O., PAPADIAS C., GARGAROPOULOS A., KONIDARIS S., KONTOGEORGI Z. \& KALAMPOKAS E. Treatment of idiopathic infertility with testosterone undecanoate a double blind study. Clin. Exp. Obst. Gyn., 1993, XX : 9-12.

25. HARGREAVE T.B., SWEETING V.M. \& ELTON R.A. Randomized trial tamoxifen versus vitamin $C$ for male infertility. Proceedings of the $12^{\text {th }}$ World Congress on Fertility and Sterility (Singapour), 1986, $9: 51-57$.

26. HOVATTA O., KOSKIMIES A., RANTA T., STENMAN U.H. \& SEPPALA M. Bromocriptine treatment of oligospermia a double blind study. Clin. Endocrinol., 1979, 11 : 377-382.

27. IACONO F., BARRA S., MONTANO L. \& LOTTI T. Intérêt de la FSH à forte dose dans le traitement de l'infertilité masculine idiopathique. J. Urol., 1996, $102: 81-84$.

28. IZZO P., CANALE D., BIANCHI B. et al. H. The treatment of male sulfertility with kallicrein. Andrologia, 1984, 16 : 156-161.

29. KECK C., BEHRE H.M., JOCKENHOVEL F. \& NIESCHLAG E. Ineffectiveness of kallicrein in treatment of male infertility: a double-blind, randomized, placebo-controlled trial. Human Reprod., 1994, 9 : 325-329.

30. KNUTH U.A., HONIGL W., BALS-PRATSCH M., SCHLEICHER G. \& NIESCHLAG E. Treatment of severe oligospermia with human chorionic gonadotropin/human menopausal gonadotropin: a placebocontrolled, double blind trial. J. Clin. Endocrinol. Metab., 1987, 65 : 1081-1087.

31. KRAUSE W., HOLLAND-MORITZ H. \& SCHRAMM P. Treatment of idiopathic oligozoospermia with tamoxifen - a randomized controlled study. Int. J. Androl., 1992, 15 : 14-18.
32. LEE K.O., NG S.C., LEE P.S. et al. Effect of growth hormone therapy in men with severe idiopathic oligospermia. Eur. J. Endocrinol., 1995, 132 : 159-162.

33. LUNGLMAYR G., MAIER U. \& SPONA J. Therapie der idiopathieschen Oligozoospermie mit Bromokriptin Resultate einer prospektive kontrollierten Studie. Andrologia, 1983, 15 : 548-553.

34. MICIC S., BILA S., ILIC V. \& SULOVIC V. Treatment of men with oligoasthenozoospermia and asthenozoospermia with kallikrein. Acta Eur. Fertil., 1985, $16: 51-53$.

35. MICIC S. \& DOTLIC R. Evaluation of sperm parameters in clinical trial with clomiphene citrate of oligospermic men. J. Urol., 1985, 133 : 211-222.

36. O'DONOVAN P.A., VANDEKERCKHOVE P., LILFORD R.J. \& HUGHES E. Treatment of male infertility: is it effective? Review and meta-analysis of published randomized controlled trials. Hum. Reprod., 1993, 8 : 1209-1222.

37. OVESEN P., JORGENSEN J.O.L., INGERSLEV J., HO K.K.Y., ORSROV H. \& CHRISTIANSEN J.S. Growth hormone treatment of subfertile males. Fertil. Steril., 1996, $66: 292-298$.

38. PAULSON D. Cortisone acetate versus clomiphene citrate in pregerminal idiopathic oligospermia. J. Urol., 1979, 121 : 432-434.

39. PRYOR J., BLANDY J., EVENS P., CHAPUT de SAINTONGE D. \& USHERWOOD M. Controlled clinical trial of arginine for infertile men with oligozoospermia. Br. J. Urol., 1978, 50 : 47-50.

40. PUSCH H.H. Oral treatment of oligozoospermia with testosterone-undecanoate: results of a double-blind placebo controlled trial. Andrologia, 1989, 21 : 76-82.

41. RADICIONI A., PARIS E., DONDERO F., BONIFACIO V. \& ISIDORI Q. Recombinant-growth hormone (rec-LGH) therapy in infertile men with idiopathic oligozoospermia. Acta Eur. Fertil., 1994, 25 : 311-317.

42. ROGERS B.O., THOMPSON T.G., GIBSON C.K., HERBERT C.M., MAX W.S. \& FARR C. The effectiveness of tamoxifen and serophene in treatment of male infertility. Am. Fertil. Soc., 1988, abstract : 609 847.

43. RONNBERG L. The effect of clomiphene citrate in different sperm parameters and serum hormone levels in preselected infertile men: a controlled double blind study. Int. J. Androl., 1980, 3 : 479-486.

44. SCHELLEN A.M.C.M. Clomiphene citrate in the treatment of male infertility. In: Bain J., Schill W.B. \& Schwarzstein L. eds. Treatment of male infertility. Springer Verlag Berlin, 1982 : 33-44.

45. SCHILL W.B. Treatment of idiopathic oligozoospermia by kallikrein: results of double blind study. Archiv. Androl., 1979, 2 : 163-170.

46. SCHILL W.B. Kinin-releasing pancreaticproteinase kallicrein. Treatment of male infertility. In: Bain J., 
Schill W.B. \& Schwarzstein L. eds. Springer Verlag Berlin, 1982 : 125-142.

47. SCHILL W.B, SCHNEIDER J. \& RING J. The use of ketotifen, a mast cell blocker, for treatment of oligoand asthenozoospermia. Andrologia, 1986, 18 : 570573.

48. SCHWARZSTEIN L. \& APARICIO N. LHRH and its analogs in the treatment of idiopathic normagonadotropic oligozoospermia. In: Bain J., Schill W., Schwarzstein L. eds. Treatment of male infertility. Springer-Verlag, Berlin, 1982 : 55-69.

49. Scottish Infertility Group: ABEL B., CARSWELL G., ELTON R. et al. Randomised trial of clomiphene citrate treatment and vitamin $\mathrm{C}$ for male infertility. Br. J. Urol., 1982, 54 : 780-784.

50. Scottish Infertility Group:HARGRAEVE T., KYLE K., BAYBY K. et al. Randomised trial of mesterolone versus vitamin $\mathbf{C}$ for male infertility. Br. J. Urol., 1984, $56: 740-744$.

51. SITERMAN S., ELTES F., WOLFSON V., ZABLUDOWSKY N. \& BARTOOV B. Effect of acupuncture on sperm parameters of males suffering from subfertility related to low sperm quality. Archives of Andrology, 1997, 39 : 155-161.

52. SOKOL R., STEINER B., BUSTILLO M., PETERSEN G. \& SWERDLOFF R. A controlled comparison of the efficacy of clomiphene citrate in male infertility. Fertil Steril. 1988, $49: 865-870$.

53. SULEIMAN S.A., ALI M.E., ZAKI Z.M.S., ELMALIK E.M.A. \& NASR M.A. Lipid peroxidation and human sperm mobility. Protective role of vitamin E. J. Androl., 1996, $17: 530-537$.

54. TOROK L. Treatment of oligozoospermia with tamoxifen (open and controlled studies). Andrologia, 1985, $17: 497-501$.

55. TOURNAYE H., VAN STEIRTEGHEM A.C. \& DEVROEY P. Pentoxifylline in idiopathic male-factor infertility: a review of its therapeutic efficacy after oral administration. Hum. Reprod., 1994, 9 : 9961000.

56. VANDERKERCKHOVE P., O'DONOVAN P.A., LILFORD R.J. \& HARADA T.W. Infertility treatment: from cookery to science. The epidemiology of randomised controlled trials. Br. J. Obstet. Gynaecol., 1993, $100: 1005-1036$.

57. VARMA T.R. \& PATEL H.R. The effect of mesterolone on sperm count, on serum follicle stimulating hormone, luteinizing hormone, plasma testosterone and outcome in idiopathic oligospermic men. Int. J. Gynecol. Obstet., 1988, 26 : 121-128.

58. VITALI G., PARENTE R. \& MELOTTI C. Carnitine supplementation in human idiopathic asthenospermia: clinical results. Drug Exp. Clin. Res., 1995, 21 : 157-159.

59. WANG G., CHAN C.W., WONG K.K. \& YEUNG K.K. Comparison of the effectiveness of placebo clomiphe- ne citrate mesterolone pentoxifylline and testosterone rebound therapy for the treatment of idiopathic oligospermia. Fertil. Steril., 1983, $40: 358-364$.

60. WHO. Task force on the diagnosis and treatment of infertility. Mesterolone and idiopathic male infertility a double blind study. Int. J. Androl., 1989, 12 : 254264.

61. WHO. A double blind trial of clomiphene citrate for the treatment of idiopathic male infertility. Int. J. Androl., 1992, $15: 299-307$.

62. WIELAND R., ANSARI A., KLEIN D., DOSHI N., HALLBERG M. \& CHEN J. Idiopathic oligospermia: control observations and response to cisclomiphene. Fertil. Steril., 1972, 23 : 471-474.

63. YAMAMOTO M., HIBI H. \& MIYAKE K. Comparison of the effectiveness of placebo and $\alpha$-blocker therapy for the treatment of idiopathic oligozoospermia. Fertil. Steril., 1995, 63 : 396-400.

64. YAMAMOTO M., HIBI H. \& MTYAKE K. New treatment of idiopathic severe oligozoospermia with mast cell blocker: results of a single blind study. Fertil. Steril., 1995, 64 : 1221-1223.

\section{ABSTRACT \\ Medical treatment of idiopathic oligoasthenozoospermia \\ A. Demoulin \\ Literature review}

In number cases, couple infertility results of an oligoasthenozoospermia without any recognized etiology. Various medical therapies have been proposed in the literature to increase sperm count and their mobility. Some studies demonstrate an improvement of spermatic parameters without taking physiological variations into consideration and without comparing the results with those obtained in patients treated with a placebo. For others, the final aim of the treatment is not specified, that means to get a pregnancy by the couples.

In this review of literature, only randomized studies giving the pregnancy rate, were taken into account. Various hormones of the hypothalamo-hypophyso-testicular axe have been used. GnRH and its analogs, gonadothropins and their purified forms, antiestrogens, androgens and aromataze inhibitors. Among these molecules, positive effects have been sporadically observed. Mesterolone stimulated spermatogenesis in one on five stu- 
dies. Testosterone undecanoate and FSH have a benefic effect on in vitro fertilization. Two antiestrogens have been tested for a couple of years. Clomiphene and tamoxifen induce a sifnificant increase in the pregnancy rates with however, some paradoxical effects. Other non hormonal molecules have also been studied. The oldest studied medication is kallicrein, a glycoprotein implicated in the release of kinins from kininogens. Contradictory results have been obtained with this therapy. Pentoxifyllin, a phosphodiesterase inhibitor is active on spermatozoa mobility in vitro, but its activity in vivo is not demostrated.

Other medicators as arginin, carnitin, mast cell blockers, $\alpha$-blockers, vitamins etc have also been tested. Publications are sporadic and any conclusion can be drawn.

In conclusion, idiopathic oligoasthenozoospermia seems still unamenable to treat. Choice of a therapy is empirical and must be leaved for trying a medically assisted treatment.

Key-words: Idiopathic male, infertility, medical treatments. 